\title{
Role of serum biomarkers and transcranial Doppler in predicting cerebral vasospasm after aneurysmal subarachnoid hemorrhage
}

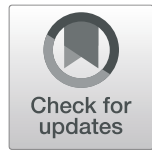

\author{
Maged Mohammad Abdelnaseer ${ }^{1}$, Ahmed Abdelkader Nemr², Sandra Mohammad Ahmed ${ }^{1}$, \\ Alshaimaa M. Aboul fotouh ${ }^{1 *}$, Amro Mohammad Ahmed Soliman², Ahmed Abdullah El-Fiki ${ }^{3}$ and \\ Safinaz Hassan Osman ${ }^{4}$
}

\begin{abstract}
Background: Aneurysmal subarachnoid hemorrhage (aSAH) can have serious consequences related to vasospasm and delayed cerebral ischemia (DCI). Serum biomarkers have emerged as a promising assessment tool to facilitate earlier diagnosis of cerebral vasospasm (CV) and to identify pre-clinical vessel narrowing.

Objectives: Our aim was to detect the predictive value of serum biomarkers such as von Willebrand factor (VWF), vascular endothelial growth factor (VEGF) and matrix metalloproteinase9 (MMP-9) in CV after aSAH.

Subjects and methods: Thirty five patients with recent aSAH were included. Patients were divided into two groups; 19 patients (CV group) and 16 patients (non-CV group). The CV group was further subdivided into 9 symptomatic (DCl) and 10 asymptomatic patients. All patients underwent transcranial Doppler (TCD) evaluations three times a week for 2 weeks measuring the mean flow velocities. Serum level of VWF, MMP-9, and VEGF were assessed twice (at onset and within 2 weeks).

Results: A statistically significant increase in serum biomarker levels was found in the CV group. Cutoff value for VWF, MMP-9, and VEGF were > $4985 \mathrm{ng} / \mathrm{ml},>495 \mathrm{ng} / \mathrm{ml}$, and > $184 \mathrm{pg} / \mathrm{ml}$, respectively. Statistically significant positive correlations were found between serum levels of biomarkers and degree of vasospasm. No difference was found in the biomarkers between symptomatic CV and asymptomatic CV.

Conclusion: Serum biomarkers are a reliable tool to predict CV following aSAH, their levels reflect the severity of vascular vasospasm, yet, they cannot predict DCI. TCD has a strong role in early detection, monitoring of post subarachnoid vasospasm and successfully capturing asymptomatic DCl.
\end{abstract}

Keywords: Aneurysmal subarachnoid hemorrhage, Cerebral vasospasm, Serum biomarkers, Transcranial Doppler

\section{Introduction}

Aneurysmal subarachnoid hemorrhage (aSAH) is a neurologic emergency caused by a brain aneurysm burst, resulting in a bleeding into the subarachinoid space. Around 10.5 per 100,000 persons per year developed subarachnoid hemorrhage (SAH) [1]. Approximately 20 to $30 \%$ of patients with aSAH suffer from delayed cerebral ischemia (DCI) due to cerebral vasospasm (CV) [2]. Cerebral vasospasm is a

\footnotetext{
* Correspondence: moyamoya15@yahoo.com

'Department of Neurology, Faculty of Medicine, Cairo University, Cairo, Egypt Full list of author information is available at the end of the article
}

heterogeneous process that has both radiographic and clinical subtypes. Radiographic CV (RCV) is a complex transient narrowing of intradural subarachnoid arteries and occur in up to $70 \%$, while clinical $\mathrm{CV}$ (CCV) occur in about $50 \%$ and it is a syndrome of neurologic deterioration related to initial aSAH [3]. A variety of different tools had been used to detect DCI such as the occurrence of focal neurological impairment, a decrease of at least 2 points on the Glasgow Coma Scale which lasts for at least $1 \mathrm{~h}$ or daily transcranial Doppler (TCD) bedside monitoring [2]. Transcranial Doppler is a recognized guideline tool for 
early detection of vasospasm [2]. Yet, the interval between TCD velocity changes and subsequent ischemia is insufficient for optimizing the benefits of medical management and early intervention [4]. Discovery of serum biomarkers in the context of SAH in relation to CV such as von Willebrand Factor (vWF), vascular endothelial growth factor (VEGF) and Matrix metalloproteinase 9 (MMP-9) have been shown to be a reliable method for predicting $\mathrm{CV}$ and improve monitoring of the response to treatment [5]. Aim of this work is to detect the role of serum vWF, MMP-9 and VEGF as a predictive value in $\mathrm{CV}$ following aSAH and to find a relationship between these biomarkers and actual occurrence and degree of $\mathrm{CV}$ and also to evaluate their advantages over clinical scales and radiological tools in predicting $\mathrm{CV}$.

\section{Subjects and methods}

This is a cross-sectional study conducted on 35 patients with recent aSAH (recruited within the first 3 days). The number of patients is calculated with sample size for frequency in population by Openepi software with confidence interval 95\%. We excluded patients with aSAH for more than $72 \mathrm{~h}$, patients who were taking anticoagulants such or antiplatelet drugs last month, patients who had blood diseases, patients who had liver and kidney dysfunction and women who were in pregnancy and lactation stages that may affect level of biomarkers.

They were selected from emergency departments of Neurology and Neurosurgery departments, Cairo University Hospitals.

The patients were divided into two groups; the first group included patients who developed cerebral vasospasm (CV group, $n=19,54.3 \%$ ) and the second group included patients who did not develop vasospasm (nonCV group, $n=16,45.7 \%$ ). The first group (patients who developed vasospasm) was further subdivided into symptomatic (DCI) group $(n=9,47.4 \%)$ and asymptomatic group $(n=10,52.6 \%)$.

All the patients were subjected to the Evaluation by World Federation of Neurosurgical Societies (WFNS) scale, Hunt and Hess $(\mathrm{H}$ and $\mathrm{H})$ scale as a clinical tool for severity assessment, and noncontrast CT brain with GE multislice 64 32 detector*2 USA 2015 HS60, Korea and graded by the modified Fisher scale as a radiological tool for severity assessment.

Evaluation and follow-up of vasospasm and VC severity were done by TCD using EZ-DOP with software version DWL2000TM Doppler, Germany GmbH (2009) three times per week till day 14. Measurements included the mean flow velocity of the middle cerebral artery and ipsilateral internal carotid artery (ICA) through the submandibular window. The Lindegaard ratio was then calculated by dividing the MFV of MCA by MFV of ICA
[6]. Patients were classified to have vasospasm if the MFV of MCA was $>120 \mathrm{~cm} / \mathrm{s}$ and the Lindegaard ratio of ipsilateral MCA and ICA was > 3, with mild-tomoderate vasospasm 3-4, moderate to sever 5-6 and sever vasospasm $\geq 6$ [6].

Two venous serum samples for measurement of vWF, VEGF and MMP-9 were collected using Diagnostic Automation Sm ELISA test kit used in ELISA DR 200D R-200Bs Microplate Reader, 2016 China; the first sample was collected on day 2 of aSAH onset and the second samples were collected at day 15 for the non-CV group, and at the time of identification of vasospasm for the $\mathrm{CV}$ group. Collected blood samples were centrifuged and the resulting supernatants were immediately frozen at $-80{ }^{\circ} \mathrm{C}$ until analysis in the ELISA Unit of Kasr $\mathrm{Al}$ Ainy University Hospital, Cairo.

\section{Statistical analysis}

Data were coded and entered using the statistical package SPSS, version 25 (SPSS Inc., Chicago, IL, USA, 2009). Data were summarized using mean and standard deviation for quantitative variables and frequencies (number of cases) and relative frequencies (percentages) for categorical variables. Comparisons between groups were done using analysis of variance (ANOVA) with multiple comparisons post hoc test or unpaired test in normally distributed quantitative variables while nonparametric Kruskal-Wallis test and Mann-Whitney test were used for non-normally distributed quantitative variables. For comparing categorical data, Chi square $\left(\chi^{2}\right)$ test was performed. Exact test was used instead when the expected frequency is less than 5 . Correlations between quantitative variables were done using Spearman correlation coefficient. Multivariate linear regression model was conducted. $p$ values less than 0.05 were considered as statistically significant.

\section{Results}

Demographic and clinical data at presentation are represented in Table 1.

TCD of the CV group shows markedly higher flow velocity at the time $\mathrm{CV}$ compared to non-CV group throughout their course. The CV group MCA MV mean was $132.15 \pm 12.4$, ICA MV was $34.05 \pm 5.28$ and Lindegaard ratio ranged from 3 to 5.7 (11 patients had mild to moderate while 8 patients had moderate to severe). In the non-CV group, the mean MCA MV was $45.44 \pm 11.97$, ICA was $29.19 \pm 4.10$ and Lindegaard ratio ranged from 1.1 to 2 .

No statistically significant relations were found between demographic and prior medical history data (age, sex, smoking, diabetes and hypertension) and WFNS or $\mathrm{H}$ and $\mathrm{H}$ grades $(p>0.05)$. Also, these data 
Table 1 Demographic, medical history, WFNS scale, Hunt and Hess scale, and modified Fisher scale in study group

\begin{tabular}{|c|c|}
\hline Demographic and medical history & Total $(n=35)$ \\
\hline \multicolumn{2}{|l|}{ Age (years) } \\
\hline Range (Mean $\pm S D$ ) & $31-80(55.60 \pm 10.95)$ \\
\hline \multicolumn{2}{|l|}{ Sex } \\
\hline Male & $17(48.6 \%)$ \\
\hline Female & $18(51.4 \%)$ \\
\hline Smoking & $7(20 \%)$ \\
\hline $\mathrm{DM}$ & $5(14.3 \%)$ \\
\hline HTN & $7(20.0 \%)$ \\
\hline WFNS scale (on admission) & Total $(n=35)$ \\
\hline GCS & $13-15(14.17 \pm 0.89)$ \\
\hline \multicolumn{2}{|l|}{ Motor deficit } \\
\hline Yes & $8(22.9 \%)$ \\
\hline No & $27(77.1 \%)$ \\
\hline \multicolumn{2}{|l|}{ Grades } \\
\hline Mild $(1,2)$ & $27(77.1 \%)$ \\
\hline Severe $(3,4)$ & $8(22.9 \%)$ \\
\hline $\mathrm{H}$ and $\mathrm{H}$ scale grades (on admission) & Total $(n=35)$ \\
\hline Mild $(1,2)$ & $20(57.1 \%)$ \\
\hline Severe $(3,4)$ & $15(42.9 \%)$ \\
\hline Modified Fischer scale grades (on admission) & Total $(n=35)$ \\
\hline 1 & $11(31.4 \%)$ \\
\hline 2 & $2(5.7 \%)$ \\
\hline 3 & $18(51.4 \%)$ \\
\hline 4 & $4(11.5 \%)$ \\
\hline CV group & 19 patients (54.3\%) \\
\hline Symptomatic CV & 9 patients (47.4\%) \\
\hline Asymptomatic CV & 10 patients (52.6\%) \\
\hline Non-CV group & 16 patients (45.7\%) \\
\hline
\end{tabular}

CV cerebral vasospasm, DM diabetes mellitus, GCS Glascow Coma Scale, $H$ and $H$ Hunt and Hess, HTN hypertension, SD standard deviation, WFNS scale World Federation of Neurosurgical Societies scale did not show a difference between the $\mathrm{CV}$ and the nonCV groups $(p>0.05)$.

No statistically significant relation was detected between WFNS, Hunt and Hess grades and occurrence of CV or DCI.

A higher proportion of patients with high grades of modified Fisher scale was found in the CV group compared to the non-CV group ( $p=0.019)$ yet no difference was found between symptomatic and asymptomatic groups.

Serum biomarkers did not differ according to sex, age, smoking, HTN or DM.

Serum concentration of vWF, VGEF and MMP-9 in the $\mathrm{CV}$ and non-CV groups in the 2nd day, were increased in a statistically significant way in the CV group (prior to occurrence of vasospasm) compared with the non-CV group ( $p<0.001$, Table 2$)$.

Correlation between Lindegaard ratio and serum biomarker concentrations of vWF, VGEF and MMP-9 in the study group shows highly significant positive correlation with $p$ value $<0.001$.

Binary Logistic Multi-Regression analysis was done for vasospasm and serum biomarker variables. It showed that serum vWF (ng/ml), VGEF (pg/ml) and MMP-9 $(\mathrm{ng} / \mathrm{ml})$ were significant predictors of vasospasm with $p$ values of $0.044,0.028$ and 0.012 , respectively.

ROC curves were constructed for serum biomarkers in the second day of aSAH as predictors of $\mathrm{CV}$ in all included patients. All serum biomarkers were significant predictors as denoted by the significantly large area under the curves (AUCs); elevated serum vWF levels of more than $4985 \mathrm{ng} / \mathrm{ml}$, MMP-9 levels of more than 495 $\mathrm{ng} / \mathrm{ml}$, and VEGF levels of more than $184 \mathrm{pg} / \mathrm{ml}$ each independently increased the odds of subsequent vasospasm (Fig. 1, Table 3).

Statistically significant positive correlation was found between Lindegaard ratio and each serum biomarker in the CV group $(p<0.001)$.

Table 2 Comparison between CV and non-CV groups in relation to serum biomarkers concentrations

\begin{tabular}{|c|c|c|c|}
\hline Serum biomarkers on day 2 & Vasospasm $(n=19)$ & No vasospasm $(n=16)$ & $\overline{p \text { value }}$ \\
\hline \multicolumn{4}{|l|}{$\mathrm{VWF}(\mathrm{ng} / \mathrm{ml})$} \\
\hline Mean \pm SD & $6613.26 \pm 1580.23$ & $4831.56 \pm 793.44$ & $<0.001$ \\
\hline Range & 4540-a9421 & 3600-a6335 & \\
\hline \multicolumn{4}{|l|}{ VEGF (pg/ml) } \\
\hline Mean \pm SD & $271.68 \pm 92.93$ & $119.41 \pm 43.05$ & $<0.001$ \\
\hline Range & $62.9-2413$ & $45.1-a 205$ & \\
\hline \multicolumn{4}{|l|}{ MMP-9 (ng/ml) } \\
\hline Mean \pm SD & $798.11 \pm 145.97$ & $339.69 \pm 91.54$ & $<0.001$ \\
\hline Range & $510-a 1000$ & $86.1-a 495$ & \\
\hline
\end{tabular}

CV cerebral vasospasm (ml), MMP-9 matrix metalloproteinase-9, $n$ number, $n g / m /$ nanogram per milliliter, $p g / m /$ picogram per milliliter, $S D$ standard deviation, VEGF vascular endothelial growth factor, VWF von Willebrand factor 


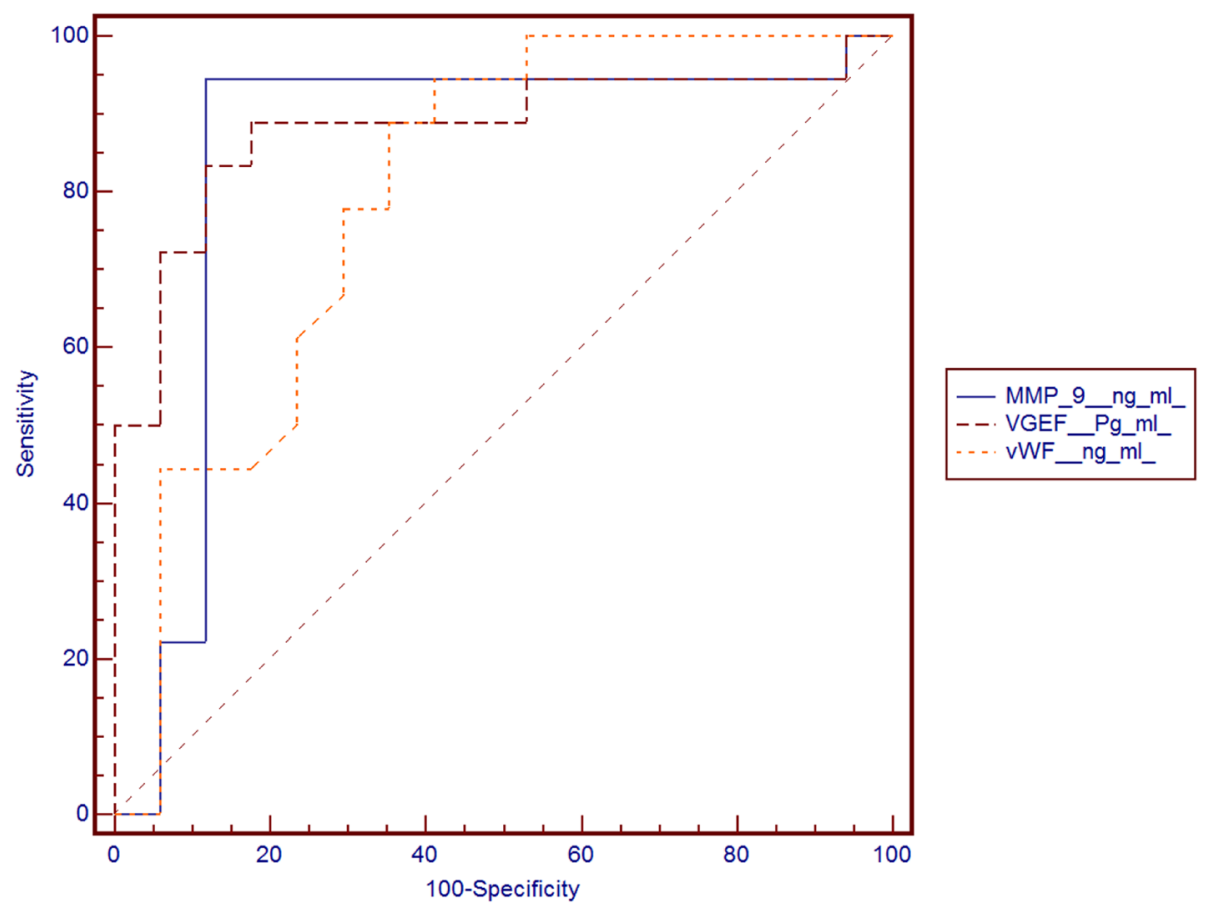

Fig. 1 ROC curve: diagnostic performance of serum biomarkers concentration in discrimination between CV and non-CV patients. Abbreviations: MMP-9 matrix metalloproteinase-9, VEGF vascular endothelial growth factor, VWF von Willebrand factor

Statistically significant increase in serum biomarkers occurred in follow-up serum samples at the time of vasospasm in the $\mathrm{CV}$ group $(p<0.05)$ compared to first sample (Table 4), unlike the non-CV group who showed no difference between the two samples $(p>0.05)$.

No statistically significant difference was found in serum biomarkers of the second sample between the symptomatic group (DCI) and non-symptomatic group $(p>0.05$, Table 4$)$.

Diagnostic performance including cut-off values, sensitivity and specificity of serum biomarker concentration are represented in Table 5.

\section{Discussion}

Prediction of vasospasm and delayed cerebral ischemia in patients with aneurysmal subarachnoid hemorrhage remains a challenge. So far, clinical predictors and imaging have offered controverted prognostic values [4]. This study showed that serum biomarkers can be predictors for $\mathrm{CV}$ yet they are less accurate in the prediction of delayed cerebral ischemia (DCI). Therefore, TCD is used as a continuous monitoring system of the $\mathrm{CBF}$ in patients with aSAH to detect early signs suggestive of $\mathrm{CV}$, and TCD can successfully capture asymptomatic DCI. In our study, age, gender, smoking, HTN and DM

Table 3 Comparison between serum biomarker concentrations on day 2 of aSAH and at time of CV

\begin{tabular}{|c|c|c|c|c|}
\hline Serum biomarkers of CV group & Day 2 & At time of detection of CV & Paired $t$ test & $p$ value \\
\hline \multicolumn{5}{|l|}{$\operatorname{VWF}(\mathrm{ng} / \mathrm{ml})$} \\
\hline Mean \pm SD & $6613.26 \pm 1580.23$ & $6901.89 \pm 1546.96$ & -2.920 & 0.009 \\
\hline Range & 4540-a9421 & 4798-a9460 & & \\
\hline \multicolumn{5}{|l|}{ VEGF (pg/ml) } \\
\hline Mean \pm SD & $271.68 \pm 92.93$ & $329.62 \pm 81.19$ & -2.047 & 0.048 \\
\hline Range & 62.9-a413 & 116-a420 & & \\
\hline \multicolumn{5}{|l|}{ MMP-9 (ng/ml) } \\
\hline Mean \pm SD & $798.11 \pm 145.97$ & $830.84 \pm 129.85$ & -4.289 & $<0.001$ \\
\hline Range & $510-\mathrm{a} 1000$ & 540-a994 & & \\
\hline
\end{tabular}

aSAH aneurysmal subarachnoid hemorrhage, $C V$ cerebral vasospasm $(\mathrm{ml}), M M P-9$ matrix metalloproteinase-9, $\mathrm{ng} / \mathrm{m} / \mathrm{nanograms}$ per milliliter, $\mathrm{pg} / \mathrm{ml}$ picogram per milliliter, SD standard deviation, VEGF vascular endothelial growth factor, vWF von Willebrand factor 
Table 4 Comparison of serum biomarkers between symptomatic CV (DCI) and asymptomatic CV groups (at vasospasm)

\begin{tabular}{|c|c|c|c|c|}
\hline \multirow[t]{2}{*}{ Serum biomarkers (day 2) } & \multirow[t]{2}{*}{ Symptomatic CV $(\mathrm{DCl}, n=9)$} & \multirow[t]{2}{*}{ Asymptomatic CV $(n=10)$} & \multicolumn{2}{|c|}{ Independent $t$ test } \\
\hline & & & $t$ & $p$ value \\
\hline \multicolumn{5}{|l|}{$\mathrm{VWF}(\mathrm{ng} / \mathrm{ml})$} \\
\hline Mean \pm SD & $6984.00 \pm 1720.65$ & $6279.60 \pm 1449.62$ & 0.938 & 0.346 \\
\hline Range & $4880-9421$ & $4540-8990$ & & \\
\hline \multicolumn{5}{|l|}{ VEGF $(\mathrm{pg} / \mathrm{ml})$} \\
\hline Mean \pm SD & $281.81 \pm 97.09$ & $262.57 \pm 93.27$ & 0.194 & 0.665 \\
\hline Range & $62.9-390.5$ & $118-413$ & & \\
\hline \multicolumn{5}{|l|}{ MMP-9 (ng/ml) } \\
\hline Mean \pm SD & $809.56 \pm 169.55$ & $787.80 \pm 129.65$ & 0.100 & 0.756 \\
\hline Range & $510-1000$ & $550-973$ & & \\
\hline
\end{tabular}

CV cerebral vasospasm, $D C l$ delayed cerebral ischemia, MMP-9 matrix metalloproteinase-9, $\mathrm{ng} / \mathrm{m} /$ nanograms per milliliter, $\mathrm{pg} / \mathrm{ml}$ picogram per milliliter, SD standard deviation, VEGF vascular endothelial growth factor, vWF von Willebrand factor

were not risk factors for the occurrence of CV. Contradictory studies have shown relation of age to $\mathrm{CV}$; one stating that it was related to younger age [7-9] and another relating it to older age [10]. Most authors have concluded that there was no correlation between the age and incidence or severity of CV [11-13]. Gender and diabetes mellitus have been rejected as well by Mijiti and colleagues (2016) as a possible cause of CV [14]. Numerous studies found that cigarette smoking or pre-existing HTN were not associated with CV $[15,16]$. Regarding clinical and radiological severity data, our results demonstrated that there was a positive correlation between the high grades of modified Fisher scale and the development of $\mathrm{CV}$, yet, we noticed no relation between $\mathrm{H}$ and $\mathrm{H}$ scale grades or WFNS grades and development of CV. Previous studies have shown different results concerning CT finding in relation to worse outcome; some showed that the higher the modified Fisher grades, the higher the incidence of CV [17-19], while others found no relation [7, 9]. Clinical scales relation to outcome have shown more consistent observations as most of the studies could not correlate initial clinical presentation of the patient to the occurrence of CV or DCI [7, 9, 20]. In this study, we investigated the association between initial concentrations of serum MMP-9, vWF and VEGF which represents markers for endothelial dysfunction with the occurrence of the CV. We also took a second sample either following vasospasm or at day 14 in patients who did not develop vasospasm to follow the serum level

Table 5 Diagnostic performance of serum biomarker concentration in discrimination of CV and non-CV patients

\begin{tabular}{lllll}
\hline Serum biomarkers (Day 2) & Cutoff & Sensitivity & Specificity & Accuracy \\
\hline $\operatorname{VWF}(\mathrm{ng} / \mathrm{ml})$ & $>4985$ & $90.68 \%$ & $65.99 \%$ & $81.29 \%$ \\
$\operatorname{VEGF}(\mathrm{pg} / \mathrm{ml})$ & $>184$ & $84.97 \%$ & $89.96 \%$ & $89.96 \%$ \\
MMP-9 $(\mathrm{ng} / \mathrm{ml})$ & $>495$ & $96.29 \%$ & $91.19 \%$ & $94.25 \%$ \\
\hline
\end{tabular}

CV cerebral vasospasm, MMP-9 matrix metalloproteinase-9, VEGF vascular endothelial growth factor, $v W F$ von Willebrand factor variability in different clinical progression. The main finding of our study was a highly statistically significant crude positive association between serum biomarker concentrations in the first sample and incidence of CV. Furthermore, we found that there was highly significant positive correlation between Lindegaard ratios with serum concentration levels of these biomarkers in all patients included in the study. The study demonstrated that early concentrations of serum MMP-9, vWF and VEGF were highly sensitive and specific for predicting subsequent CV with MMP-9 being the most significant predictor. Yet, no difference was found between patients who developed DCI and those who did not. This finding rejects the fact that the biomarkers were related to ischemic stroke [4]. The biomarker serum levels were not affected by demographic, medical history or clinical scales. Also, these biomarkers had low statistically significant correlation with modified Fisher scale grades which suggest that these biomarkers may be an independent predictor of $\mathrm{CV}$. Our results were in accordance with many studies that confirmed the relation of the serum levels of MMP-9 [21] and vWF levels [22] to the occurrence of the $\mathrm{CV}$ group compared to the non-CV group.

In the current study, cutoff values showed that elevated serum levels of more than $4985 \mathrm{ng} / \mathrm{ml}, 495 \mathrm{ng} / \mathrm{ml}$ and 184 $\mathrm{pg} / \mathrm{ml}$ in vWF, MMP-9 and VEGF, respectively independently increase the odds of subsequent CV. McGrit and colleagues (2002) reported that elevated plasma vWF levels > 5,500 ng/ml, MMP-9 levels > $700 \mathrm{ng} / \mathrm{ml}$ and VEGF levels $>0.12 \mathrm{ng} / \mathrm{ml}$ each independently increased the odds of CV [4]. We, thus, recommend that every institution must have their own reference cutoff value. The serum levels of these biomarkers kept increasing in the second sample in patients who developed CV unlike those who did not have CV. The results of this study also showed that the serum concentrations of these biomarkers in the non-CV group did not increase or decrease significantly along with time, so we considered level of serum 
biomarkers in the non-CV patients (as control) a reference value. These findings suggest that these markers may be specific for the arterial changes associated with CV. Yet, absence of difference between patients who developed delayed cerebral ischemia (DCI) and those who did not exclude the possibility of relation between these biomarkers and ischemic sequelae as stated before [22] making them specific for vasospasm. Doppler ultrasound findings showed a highly significant positive correlation between the Lindegaard ratio (reflecting degree of vasospasm) and serum concentration level of each one of the biomarkers in all patients irrespective of CV. This finding confirms that these biomarkers may play a role in the pathophysiological mechanism of vasoconstriction, yet, that the development of $\mathrm{CV}$ begins when these biomarkers cross a certain threshold level.

\section{Conclusion}

TCD has a significant role in early detection, monitoring of post subarachnoid vasospasm and successfully capturing asymptomatic DCI. VEGF, MMP-9 and vWF are strong independent predictors of vasospasm in patients with aSAH, yet, they cannot predict delayed cerebral ischemia. These biomarkers show better predictive value for CV compared to patient's initial clinical presentation and brain imaging.

\section{Abbreviations \\ ANOVA: Analysis of variance; aSAH: Aneurysmal subarachnoid hemorrhage; CCV: Clinical CV; CT: Computed tomography; CTA: CT angiography; $\mathrm{CV}$ : Cerebral vasospasm; DIC: Delayed cerebral ischemia; $\mathrm{H}$ and $\mathrm{H}$ : Hunt and Hess; HTN: Hypertension; ICA: Internal carotid artery; IQR: Interquartile range; MCA: Middle cerebral artery; MFV: Mean flow velocity; MMP-9: Matrix metalloproteinase 9; RCV: Radiographic CV; ROC: Receiver operating characteristic; TCD: Transcranial Doppler; VEGF: Vascular endothelial growth factor; vWF: von Willebrand factor; WFNS: World Federation of Neurosurgical Societies}

\section{Acknowledgements}

Not applicable

\section{Authors' contributions}

MMA participated in the concept and the study design. AAN applied the inclusion and exclusion criteria and drafted the manuscript. SMA participated in the concept and design of the study. AMA participated in the concept and design of the study and is responsible for the laboratory handling, preparation, and analysis of the blood samples. AMAS participated in the design of the study and analysis of data, helped in the clinical examination, helped in applying the inclusion and exclusion criteria, and performed the statistical analysis. AAE is responsible for the coordination and recruitment of participants. SHO helped in the clinical examination and data analysis. All authors read and approved the final manuscript.

\section{Funding}

This research received no specific grant from any funding agency in the public, commercial, or not-for-profit sectors.

\section{Availability of data and materials}

The datasets generated and/or analyzed during the current study are not publicly available due to the current Cairo University regulations and Egyptian legislation but are available from the corresponding author on reasonable request and after institutional approval.

\section{Ethics approval and consent to participate}

Informed written consent was taken from each patient. All data obtained from every patient were confidential and were not used outside the study. The patients have the rights to withdraw from the study at any time without giving any reason. All the cost of the investigations was afforded by the researcher. Our study was approved by the ethical committee of the Department of Neurology, Faculty of Medicine, Cairo University, on 5th of May 2017, but Cairo University does not provide the approval reference number.

\section{Consent for publication}

Not applicable

\section{Competing interests}

The authors declare that they have no competing interests.

\section{Author details}

${ }^{1}$ Department of Neurology, Faculty of Medicine, Cairo University, Cairo, Egypt. ${ }^{2}$ Department of Neurology, Faculty of Medicine, Military Academy, Cairo, Egypt. ${ }^{3}$ Department of Neurosurgery, Faculty of Medicine, Cairo University, Cairo, Egypt. ${ }^{4}$ Department of Anesthesia, Faculty of Medicine, Cairo University, Cairo, Egypt.

Received: 20 August 2019 Accepted: 23 January 2020

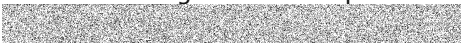

\section{References}

1. Grasso G, Alafaci C, Macdonald RL. Management of aneurysmal subarachnoid hemorrhage: state of the art and future perspectives. Surg Neurol Int. 2017:8:11.

2. Vergouwen MD, Vermeulen M, van Gijn J, Rinkel GJ, Wijdicks EF, Muizelaar $J$ J, et al. Definition of delayed cerebral ischemia after aneurysmal subarachnoid hemorrhage as an outcome event in clinical trials and observational studies: proposal of a multidisciplinary research group. Stroke. 2010;41(10):2391-5.

3. Velat GJ, Kimball MM, Mocco JD, Hoh BL. Vasospasm after aneurysmal subarachnoid hemorrhage: review of randomized controlled trials and meta-analyses in the literature. World Neurosurg. 2011;76(5):446-54.

4. McGirt MJ, Lynch JR, Blessing R, Warner DS, Friedman AH, Laskowitz DT. Serum von Willebrand factor, matrix metalloproteinase-9, and vascular endothelial growth factor levels predict the onset of cerebral vasospasm after aneurysmal subarachnoid hemorrhage. Neurosurgery. 2002;51(5):1128-34.

5. Przybycien-Szymanska MM, Ashley WW Jr. Biomarker discovery in cerebral vasospasm after aneurysmal subarachnoid hemorrhage. J Stroke Cerebrovasc Dis. 2015;24(7):1453-64.

6. Gonzalez NR, Boscardin WJ, Glenn T, Vinuela F, Martin NA. Vasospasm probability index: a combination of transcranial Doppler velocities, cerebral blood flow, and clinical risk factors to predict cerebral vasospasm after aneurysmal subarachnoid hemorrhage. J Neurosurg. 2007;107(6):1101-12.

7. Kale SP, Edgell RC, Alshekhlee A, Borhani Haghighi A, Sweeny J, Felton J, et al. Age-associated vasospasm in aneurysmal subarachnoid hemorrhage. J Stroke Cerebrovasc Dis. 2013;22(1):22-7.

8. Malinova V, Schatlo B, Voit M, Suntheim $P$, Rohde $V$, Mielke D. Identification of specific age groups with a high risk for developing cerebral vasospasm after aneurysmal subarachnoid hemorrhage. Neurosurg Rev. 2016;39(3):429-36.

9. Arias $M R$, Pacheco $C D$, Agudelo $Y$. Risk factors for cerebral vasospasm in patients with spontaneous subarachnoid hemorrhage. Rev Colomb Radiol. 2017;28(2):4657-62.

10. de Rooij NK, Linn FH, van der Plas JA, Algra A, Rinkel GJ. Incidence of subarachnoid haemorrhage: a systematic review with emphasis on region, age, gender and time trends. J Neurol Neurosurg Psychiatry. 2007;78(12): 1365-72.

11. Jung SW, Lee CY, Yim MB. The relationship between subarachnoid hemorrhage volume and development of cerebral vasospasm. J Cerebrovasc Endovasc Neurosurg. 2012 Sep;14(3):186-91.

12. Ryttlefors M, Enblad P, Ronne-Engström E, Persson L, llodigwe D, Macdonald RL. Patient age and vasospasm after subarachnoid hemorrhage. Neurosurgery. 2010;67(4):9117. 
13. Inagawa T, Yahara K, Ohbayashi N. Risk factors associated with cerebral vasospam following aneurysmal subarachnoid hemorrhage. Neurol Med Chir. 2014;54(6):465-73.

14. Mijiti M, Mijiti P, Axier A, Amuti M, Guohua Z, Xiaojiang C, et al. Incidence and predictors of angiographic vasospasm, symptomatic vasospasm and cerebral infarction in Chinese patients with aneurysmal subarachnoid hemorrhage. PLoS One. 2016;11(12):e0168657.

15. Badjatia N, Topcuoglu MA, Buonanno FS, Smith EE, Nogueira RG, Rordorf $\mathrm{GA}$, et al. Relationship between hyperglycemia and symptomatic vasospasm after subarachnoid hemorrhage. Crit Care Med. 2005;33(7):1603-9.

16. Dupont SA, Wijdicks EF, Manno EM, Lanzino G, Rabinstein AA. Prediction of angiographic vasospasm after aneurysmal subarachnoid hemorrhage: value of the Hijdra sum scoring system. Neurocrit Care. 2009;11(2):172-6.

17. Eagles ME, Jaja BNR, Macdonald RL. Incorporating a modified Graeb Score to the modified Fisher scale for improved riskv prediction of delayed cerebral ischemia following aneurysmal subarachnoid hemorrhage. Neurosurgery. 2018;82(3):299305.

18. Platz J, Güresir E, Wagner M, Seifert V, Konczalla J. Increased risk of delayed cerebral ischemia in subarachnoid hemorrhage patients with additional intracerebral hematoma. J Neurosurg. 2017;126(2):504-10.

19. Griessenauer CJ, Starke RM, Foreman PM, Hendrix P, Harrigan MR, Fisher WS, et al. Associations between endothelin polymorphisms and aneurysmal subarachnoid hemorrhage, clinical vasospasm, delayed cerebral ischemia, and functional outcome. J Neurosurg. 2018;128(5):1311-7.

20. Smith ML, Abrahams JM, Chandela S, Smith MJ, Hurst RW, Le Roux PD Subarachnoid hemorrhage on computed tomography scanning and the development of cerebral vasospasm: the Fisher grade revisited. Surg Neurol. 2005;63(3):229-34.

21. Wang L, Gao Z. Expression of MMP-9 and IL 6 in patients with subarachnoid hemorrhage and the clinical significance. Exp Ther Med. 2018;15(2):1510-4.

22. Li WH, Hui CJ, Ju H. Expression and significance of VWF, GMP-140 and ADAMTS13 in patients with aneurysmal subarachnoid hemorrhage. Eur Rev Med Pharmacol Sci. 2017;21(19):4350-6.

\section{Publisher's Note}

Springer Nature remains neutral with regard to jurisdictional claims in published maps and institutional affiliations.

\section{Submit your manuscript to a SpringerOpen ${ }^{\circ}$ journal and benefit from:}

- Convenient online submission

- Rigorous peer review

- Open access: articles freely available online

- High visibility within the field

- Retaining the copyright to your article

Submit your next manuscript at $\boldsymbol{\nabla}$ springeropen.com 